\title{
Uncharacteristic Task-Evoked Pupillary Responses Implicate Atypical Locus Ceruleus Activity in Autism
}

\author{
${ }^{\circledR}$ Michael C. Granovetter, ${ }^{1,2,3}{ }^{\circledR}$ Charlie S. Burlingham, ${ }^{4}{ }^{\circledR}$ Nicholas M. Blauch, ${ }^{2}{ }^{\circledR}$ Nancy J. Minshew, ${ }^{5}$ \\ ${ }^{-}$David J. Heeger, ${ }^{4,6}$ and ${ }^{\circledR}$ Marlene Behrmann ${ }^{1,2}$ \\ ${ }^{1}$ Department of Psychology, Carnegie Mellon University, Pittsburgh, Pennsylvania $15213,{ }^{2}$ Neuroscience Institute, Carnegie Mellon University, \\ Pittsburgh, Pennsylvania 15213, ${ }^{3}$ School of Medicine, University of Pittsburgh, Pittsburgh, Pennsylvania 15213, ${ }^{4}$ Department of Psychology, New \\ York University, New York, New York 10003, ${ }^{5}$ Department of Psychiatry, University of Pittsburgh, Pittsburgh, Pennsylvania 15213, and ${ }^{6}$ Center for \\ Neural Science, New York University, New York, New York 10003
}

Autism spectrum disorder (ASD) is characterized partly by atypical attentional engagement, reflected in exaggerated and variable responses to sensory stimuli. Attentional engagement is known to be regulated by the locus ceruleus (LC). Moderate baseline LC activity globally dampens neural responsivity and is associated with adaptive deployment and narrowing of attention to task-relevant stimuli. In contrast, increased baseline LC activity enhances neural responsivity across cortex and widening of attention to environmental stimuli regardless of their task relevance. Given attentional atypicalities in ASD, this study is the first to evaluate whether, under different attentional task demands, individuals with ASD exhibit a different profile of LC activity compared with typically developing controls. Males and females with ASD and age- and gender-matched controls participated in a one-back letter detection test while task-evoked pupillary responses, an established correlate for LC activity, were recorded. Participants completed this task in two conditions, either in the absence or presence of distractor auditory tones. Compared with controls, individuals with ASD evinced atypical pupillary responses in the presence versus absence of distractors. Notably, this atypical pupillary profile was evident despite the fact that both groups exhibited equivalent task performance. Moreover, between-group differences in pupillary responses were observed specifically in response to task-relevant events, providing confirmation that the group differences most likely were specifically associated with distinctions in LC activity. These findings suggest that individuals with ASD show atypical modulation of LC activity with changes in attentional demands, offering a possible mechanistic and neurobiological account for attentional atypicalities in ASD.

Key words: attention; autism; excitation-inhibition; locus ceruleus; neural gain; pupillometry

Significance Statement

Individuals with autism spectrum disorder (ASD) exhibit atypical attentional behaviors, including altered sensory responses and atypical fixedness, but the neural mechanism underlying these behaviors remains elusive. One candidate mechanism is atypical locus ceruleus (LC) activity, as the LC plays a critical role in attentional modulation. Specifically, LC activity is involved in regulating the trade-off between environmental exploration and focused attention. This study shows that, under tightly controlled conditions, task-evoked pupil responses, an LC activity proxy, are lower in individuals with ASD than in controls, but only in the presence of task-irrelevant stimuli. This suggests that individuals with ASD evince atypical modulation of LC activity in accordance with changes in attentional demands, offering a mechanistic account for attentional atypicalities in ASD.

Received Nov. 13, 2019; revised Feb. 7, 2020; accepted Feb. 29, 2020.

Author contributions: M.C.G., D.J.H., and M.B. designed research; M.C.G. and N.J.M. performed research; M.C.G., C.S.B., and N.M.B. analyzed data; M.C.G. wrote the first draft of the paper; M.C.G., C.S.B., N.M.B., D.J.H., and M.B. edited the paper.

The authors declare no competing financial interests.

This work was supported by National Institute of General Medical Sciences Awards T32GM008208 and T32GM081760 to M.C.G., and Simons Foundation Autism Research Initiative Grant to M.B. and D.J.H. The content is solely the responsibility of the authors and does not necessarily represent the official view of the National Institute of General Medical Sciences or the National Institutes of Health. We thank Drs. Yael Niv and Eran Eldar for initial discussions that stimulated the early framework of this study; Dr. Shaun E. Eack, Patricia J. McCarroll, and Michelle Perrin for assisting with recruitment; John J. Markiewicz for organization of the neuropsychological testing data; Drs. Ilan Dinstein and Eran Eldar for input on the study design; Drs. Ilan Dinstein and Sarah M. Haigh, whose experiment code was adapted for the study task; Dr. Timothy Verstynen and Madhumita Harish for input on the analyses; and the participants for making this research possible.

Correspondence should be addressed to Michael C. Granovetter at granovetter@cmu.edu.

https://doi.org/10.1523/JNEUROSCI.2680-19.2020

Copyright $\odot 2020$ the authors

\section{Introduction}

Autism spectrum disorder (ASD) is a neurodevelopmental condition characterized by atypicalities in social, sensory, and motor behaviors, with unclear neural underpinnings (Lord et al., 2018). The diversity of cognitive behaviors implicated in ASD suggests a possible global disruption in the homeostasis of excitatoryinhibitory (E-I) neural activity (Sur and Rubenstein, 2005; Robertson et al., 2013; Dinstein et al., 2015; Rosenberg et al., 2015). Specifically, an inability to modulate neural gain, the likelihood of excitatory versus inhibitory output from a given input (Servan-Schreiber et al., 1990), could result in increased variability in neural responsivity (Rosenberg et al., 2015). Consistent with this account, fMRI studies have demonstrated that 
individuals with ASD exhibit higher intraindividual variability of stimulus-evoked hemodynamic responses in sensory cortical areas compared with controls (Dinstein et al., 2012; Haigh et al., 2015). This neural variability may be related to, or be a product of, an inability to regulate neural gain globally.

The locus ceruleus (LC) globally regulates neural gain in association with cognitive task engagement (i.e., deployment of attention to task-relevant vs distractor stimuli) (Aston-Jones and Cohen, 2005; Eldar et al., 2013). With moderate tonic (baseline) LC activity, phasic responses can be elicited specifically in association with decisions executed on a task, and this mode of activity correlates with increased task engagement. However, with higher tonic LC activity, phasic responses in association with decision processes are weaker, a mode of activity correlating with decreased task engagement and increased distractibility (Aston-Jones and Cohen, 2005; Gilzenrat et al., 2010). Furthermore, with high tonic (and low phasic) LC activity, neural gain is increased throughout cortex, such that neural responsivity is arbitrarily and globally elevated (Aston-Jones and Cohen, 2005).

If individuals with ASD were to exhibit lower phasic, and higher tonic, LC activity than controls, with consequent increased neural sensitivity throughout cortex (Aston-Jones and Cohen, 2005; Eldar et al., 2013), this might explain the unreliability of neural responses to sensory stimuli in individuals with ASD (Dinstein et al., 2012; Haigh et al., 2015). Individuals with ASD are indeed known to exhibit elevated tonic pupil sizes (Anderson and Colombo, 2009; Anderson et al., 2013; Blaser et al., 2014), and pupil size has been shown to correlate with LC activity in nonhuman primates (Aston-Jones et al., 1994; Joshi et al., 2016). Despite the multiplicity of provocative findings, however, no study has clearly demonstrated whether individuals with ASD evince an atypical LC profile under different attentional demands. A further desideratum of such a study would be to demonstrate differences in LC profiles when behavioral performance is comparable between ASD participants and controls. As pupillary responses can be attributed to a number of processes (Larsen and Waters, 2018), such an outcome would reveal an inherent alteration in LC activity rather than any physiological differences that might instead be a direct consequence of differences in behavior across the groups.

This study examines whether individuals with ASD exhibit differences in phasic LC activity compared with typically developing controls under different attentional demands, by exploiting phasic pupillary responses as a signature of LC activity. The phasic pupillary response to task decisions is an appropriate readout of LC activity because it is specifically associated with LC-mediated processing and allows for between-group comparisons not confounded by unrelated individual differences in pupil size (AstonJones and Cohen, 2005; Eldar et al., 2013). Here, adults with and without ASD performed a one-back letter detection task either in the absence or presence of auditory distractors. Typically developing individuals, who can flexibly modulate LC activity in the context of attentional demands, might be expected to exhibit greater task-evoked pupillary responses in the presence versus absence of distractors. If individuals with ASD were to demonstrate consistently lower phasic (and higher tonic) LC activity, task-relevant phasic pupillary responses would be expected to be reduced relative to controls' but only in the presence versus absence of distractors and not adapted to the specifics of the task conditions.

\section{Materials and Methods}

Subject details

Twenty-six individuals with ASD and 26 age- and gender-matched controls were initially recruited and participated. Three individuals with
Table 1. Clinical metrics of participants with ASD ${ }^{\mathrm{a}}$

\begin{tabular}{lrrcr}
\hline & Mean & SD & Minimum & Maximum \\
\hline ADOS-LaC0 & 3.78 & 1.44 & 2 & 8 \\
ADOS-RSI & 7.17 & 2.06 & 4 & 12 \\
ADOS-RRB & 1.70 & 1.64 & 0 & 6 \\
VIQ & 112.57 & 12.63 & 91 & 141 \\
PIQ & 110.70 & 17.61 & 81 & 134 \\
FSIQ & 113.83 & 15.02 & 86 & 134 \\
\hline
\end{tabular}

${ }^{a} \mathrm{VIQ}$, Verbal Intelligence Quotient; PIQ, Performance Intelligence Quotient; FSIQ, Full Scale IQ.

ASD and two controls were not included in the data analyses because they did not complete both experimental task conditions $(n=3$ participants with ASD, $n=1$ control) or because their data were discarded based on artifacts in the data and/or excessive blinks described below ( $n=1$ control). Thus, a total of 23 individuals with ASD and 24 controls were ultimately examined in this study. The diagnosis of participants with ASD was confirmed by an expert clinician at the Center for Excellence in Autism Research at the University of Pittsburgh, and controls were recruited from the local Pittsburgh community. Descriptive statistics on the Autism Diagnostic Observation Schedule (ADOS) (Lord et al., 1989) and Wechsler Abbreviated Scale of Intelligence (Kaufman and Lichtenberger, 1999) for participants with ASD, who were included in the analyses, are described in Table 1.

In recruitment, groups were matched by age, gender, and handedness (confirmed with the Edinburgh Handedness Inventory) (Oldfield, 1971). To determine whether these characteristics were comparable between groups, a logistic regression model to predict group membership was fitted with these features as predictors. Group could not be predicted from a participant's age $(z=1.63, p=0.10)$, gender $(z=0.05$, $p=0.96)$, or handedness $(z=0.16, p=0.88)$, indicating comparability of the groups on these variables. See Tables 2 and 3 for descriptive statistics of these characteristics for participants whose data were included in the analyses.

Participants completed questions about additional variables that might affect pupillometry measurements. As caffeine intake can affect pupil size (Abokyi et al., 2017), participants were asked about their caffeine intake on the day of the study session. Participants also listed the medications they were taking, and the UpToDate database (Wolters Kluwer) was used to determine which, if any, medications interact with the adrenergic system (Table 4). Finally, whether a participant was wearing eyeglasses was noted as this could potentially affect pupillometry recordings. A logistic regression model to predict group was fitted with these features as predictors. Group membership was predicted by use of adrenergic-related medication $(z=3.16, p<0.01)$, but not by caffeine intake $(z=1.37, p=0.17)$ or wearing eyeglasses $(z=0.16, p=0.87$; Table $3)$. Indeed, more than half of ASD participants reported taking adrenergic-related medication, while only a single control reported doing so. The possibility of medication use confounding effects observed on group differences in behavior or pupillary responses was thus explored in the analyses described below. As there were no group differences in age, gender, handedness, caffeine use, or eyeglasses, the effects of these descriptive variables on dependent measures were not tested to avoid overfitting regression models (Babyak, 2004) or obtaining false positives from multiple comparisons testing (Ranganathan et al., 2016).

The Carnegie Mellon University Institutional Review Board reviewed and approved this research, and all participants provided informed consent.

\section{Experimental design and statistical analyses}

Task design. Participants' heads were positioned in a chinrest at a distance of $\sim 60 \mathrm{~cm}$ from an $\sim 38 \times 31 \mathrm{~cm}$ computer monitor. The luminance and contrast settings of the monitor, as well as the ambient lighting in the room, were approximately constant throughout the experimental session and across participants. Task stimuli were presented using the Psychophysics Toolbox (Brainard, 1997) in MATLAB (The MathWorks), and participants completed two versions of the task: without and with accompanying distractors. 
Table 2. Age and handedness of participants by group ${ }^{a}$

\begin{tabular}{|c|c|c|c|c|c|c|c|c|}
\hline & ASD (mean) & Control (mean) & ASD (SD) & Control (SD) & ASD (minimum) & Control (minimum) & ASD (maximum) & Control (maximum) \\
\hline Age (yr) & 32.04 & 28.38 & 8.12 & 6.74 & 21 & 21 & 49 & 47 \\
\hline Handedness (EHI) & 60.84 & 59.16 & 50.23 & 45.41 & -70.00 & -85.71 & 100.00 & 100.00 \\
\hline
\end{tabular}

${ }^{a_{E}}$ HI, Edinburgh Handedness Inventory. It ranges from -100 (left-handed dominance) to 100 (right-handed dominance).

Table 3. Percentages of participants (by group) who were female, had consumed caffeine on the day of the study session, were currently taking medications that interact with the adrenergic system, and wore eyeglasses

\begin{tabular}{lcc}
\hline & ASD (\%) & Control (\%) \\
\hline Female & 8.70 & 8.33 \\
Caffeine $^{\prime}$ & 43.48 & 54.17 \\
Adrenergic medication(s) $^{\mathrm{a}}$ & 56.52 & 4.17 \\
Eyeglasses & 52.17 & 41.67 \\
\hline
\end{tabular}

${ }^{a}$ Significant predictor of group.

Table 4. Medications that participants reported taking

\begin{tabular}{|c|c|}
\hline Medication & Participant identification $\operatorname{codes}^{a}$ \\
\hline \multicolumn{2}{|c|}{ Medications known to interact with the adrenergic system } \\
\hline Aripiprazole & $\mathrm{A} 1, \mathrm{~A} 2$ \\
\hline Atenolol & A3 \\
\hline Atomoxetine & A4 \\
\hline Bupropion & A1 \\
\hline Citalopram & A5 \\
\hline Escitalopram & $A 2, A 3$ \\
\hline Fluvoxamine & A6 \\
\hline Lisdexamfetamine & A1 \\
\hline Lisinopril & $A 1, A 7$ \\
\hline Methylphenidate & $\mathrm{A} 8, \mathrm{A9}, \mathrm{A} 10$ \\
\hline Propranolol & A11 \\
\hline Quetiapine & A10 \\
\hline Sertraline & $A 4, A 10, A 11, A 12, A 13$ \\
\hline Tranylcypromine & A14 \\
\hline Trazodone & $\mathrm{A} 15, \mathrm{C} 1$ \\
\hline \multicolumn{2}{|c|}{ Medications not known to interact with the adrenergic system } \\
\hline Cetirizine & A1 \\
\hline Dimethylglycine & A5 \\
\hline Docusate & $\mathrm{A} 1$ \\
\hline Ethinyl estradiol and drospirenone & A7 \\
\hline Fexofenadine & A12 \\
\hline Lamotrigine & A1 \\
\hline Levothyroxine & A11 \\
\hline Loratadine & $A 2, A 15, A 16$ \\
\hline Lorazepam & A15 \\
\hline Melatonin & $\mathrm{A} 4, \mathrm{~A} 15$ \\
\hline Mesalamine & A17 \\
\hline Metformin & $A 2, A 18$ \\
\hline Pantoprazole & A15 \\
\hline Paroxetine & A18 \\
\hline Pramipexole & $\mathrm{C} 2$ \\
\hline Ranitidine & $\mathrm{A} 1$ \\
\hline Simvastatin & A5 \\
\hline Terbinafine & A19 \\
\hline Topiramate & $\mathrm{A} 1$ \\
\hline Zolpidem & A4 \\
\hline
\end{tabular}

${ }^{a} \mathrm{~A}$, Autism; C, control.

The luminance of all stimuli was comparable to the background: specifically, the $L^{*}$ value of the CIELAB color space (McGuire, 1992) was approximately equal for all colors in the task display. On a gray $($ CIELAB $=[5776.9,0,0])$ background, participants viewed a green $(\mathrm{CIELAB}=[5777,-4812.8,4645.1])$ circle positioned at the center of

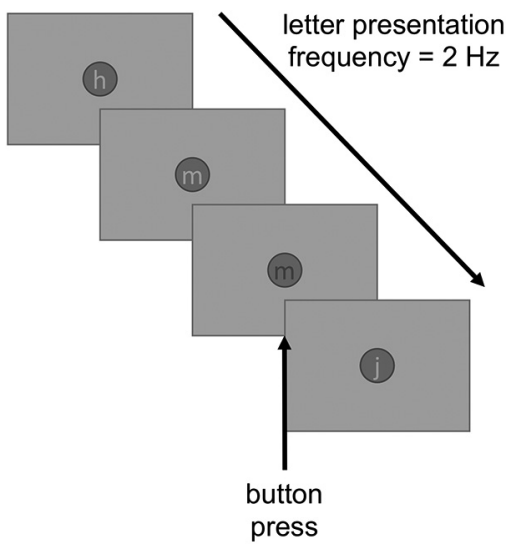

Figure 1. Schematic of the one-back letter detection task. Participants viewed individual presentations of letters at a rate of $2 \mathrm{~Hz}$ and were instructed to press a button on observing a consecutive letter repeat. For a duration of $0.5 \mathrm{~s}$, letters became purple or red in response to a correct or incorrect button press, respectively. The visual display was isoluminant throughout the task session. In the first half of the experiment, participants performed the task in the absence of distractor stimuli. In the second half, participants were exposed to series of tones played temporally independent of the task sequence.

the screen. A set of 15 lowercase gray (CIELAB $=[5776.9,0,0])$ letters randomly appeared one at a time at a frequency of $2 \mathrm{~Hz}$ within the circle. Participants were instructed to indicate, using a keyboard press, each instance in which a consecutive letter repeat occurred. To provide feedback to participants, when a key was pressed in the second following a consecutive letter repetition, the letter on display became purple $(\mathrm{CIELAB}=[5772.4,3020.8,-5570])$ for a $0.5 \mathrm{~s}$ duration subsequent to the key press. For all other key presses, the letters became red (CIELAB $=[5780.3,5857.9,5501.7])$ for a $0.5 \mathrm{~s}$ duration subsequent to the key press (Fig. 1). While letter presentation was random, a pair of consecutive letters would not repeat within a $6 \mathrm{~s}$ interval. The letters were presented in the same order to all participants; and out of a total of $\sim 1584$ letter presentations, there were 54 total consecutive letter repetitions. This constituted the no-distractor condition.

Participants then completed the same task, but this time in the presence of distractor auditory stimuli, following a task design adapted from prior studies (Dinstein et al., 2012; Haigh et al., 2015). As the participants performed the same letter-repeat task, eleven $600 \mathrm{~Hz}$ tones were played through a headset, with each tone lasting for $0.15 \mathrm{~s}$, with $0.15 \mathrm{~s}$ intervals between tones. Initiation of the 11 tones was separated by a random intertrial interval, ranging between 6 and $10 \mathrm{~s}$ to prevent participants from predicting the onset of the tones, and the timing of tone onsets was not associated with letter presentations or repeats. During this block, a total of $\sim 1607$ letters were presented, with 65 total consecutive letter repetitions.

Each of the two task conditions consisted of three blocks of letter presentations, with breaks in-between.

Eye-tracking. Pupil area and coordinates were measured with the EyeLink 1000 (SR Research; http://www.sr-research.com/) at a sampling rate of $1000 \mathrm{~Hz}$. The eye-tracker was positioned below the computer monitor and was angled to record measurements from a single eye. A 3 or 5 point display grid was used for calibration, conducted before each experimental block. Thresholds for pupil detection were adapted for each participant due to individual differences between participants, such as participants' need to wear glasses or contact lenses, eye color, and eye 
size. To determine whether these parameters of the eye-tracker were comparable between the groups, a logistic regression model to predict group was fitted with the thresholds for pupil and cornea detection as predictors. Neither pupil detection threshold (ASD: mean $=81.30$, $\mathrm{SD}=8.29$; Control: mean $=77.29, \mathrm{SD}=9.09 ; z=1.47, p=0.14$ ) nor cornea detection threshold ( $\mathrm{ASD}$ : mean $=250.43, \mathrm{SD}=1.44$; Control: mean $=250.00, \mathrm{SD}=0 ; z=0.01 p=0.99$ ) was predictive of group.

The pupillometry data were preprocessed using custom in-house scripts in MATLAB version 9.5.0 (The MathWorks), as well as adapted blink/artifact interpolation code (Urai et al., 2017). Pupil area was converted to pupil diameter, taking into account the fact that the eye-tracker used a centroid-fitting model in detecting the pupil. Instances in which the eye-tracker could not track the pupil and instances in which the pupil size was beyond $3 \mathrm{SD}$ from the median pupil size of the block were considered to be artifacts. During blinks and artifacts (including those detected by the EyeLink 1000 software), the data were linearly interpolated over these intervals, and nearest neighbor interpolation was used at the start and endpoints of these intervals. Blinks, partial blinks, or other artifacts detected within $0.25 \mathrm{~s}$ of one another were linearly interpolated as a single blink, and data were linearly interpolated from $0.15 \mathrm{~s}$ before and $0.15 \mathrm{~s}$ after each detected blink. Nearest neighbor interpolation was used at the start and end of each blink/artifact. To interpolate over peakdetected blinks, the pupil size data were initially smoothed using a twodimensional digital filter with an 11 point symmetric Hann window. Peak-detected blinks (separated in time by a minimum duration of $0.5 \mathrm{~s}$ ) were subsequently interpolated: peak-detected blinks detected within $0.25 \mathrm{~s}$ of one another were interpolated as a single peak-detected blink, and data were interpolated from $0.3 \mathrm{~s}$ before and $0.15 \mathrm{~s}$ subsequent to each peak-detected blink. Nearest neighbor interpolation was also used at the start and end of each peak-detected blink. Furthermore, to meet criteria for inclusion in the study, a participant's data were excluded if blinks or artifacts constituted more than two-thirds of the duration of an experimental condition (absence vs presence of distractors) across all blocks for that condition. Only one participant, a control, did not meet this criterion, and his data are not included in the summary statistics above nor in the analyses below.

To assess each participant's baseline pupil size, at the start of each block, participants viewed a central fixation (the same green circle on a gray background used in the experiment) for $\sim 45 \mathrm{~s}$ before starting the task. For each participant, the median of all pupil size measurements across these passive viewing periods was computed. One participant (in the ASD group) blinked and exhibited artifacts for more than two-thirds of the duration of baseline pupil size recordings. This participant's data were thus discarded from analyses of baseline pupil size only.

Parameters for preprocessing of the pupillometry data were decided on before completion of data collection and final performance of statistical analyses, based on visual inspection of initial data collection. For analyses of task-evoked pupil responses, pupil-size measurements were converted to percent signal change relative to the mean pupil size within the entire block in which they were collected. This was done to normalize between-block differences in pupil response amplitudes caused by interaction between the tonic and phasic components of the pupil signal (Eldar et al., 2013). To eliminate very low frequency fluctuations, the pupil size signal was high-pass filtered with a Butterworth filter of order 4 with a cutoff of $0.03 \mathrm{~Hz}$. To reduce the sampling rate of the signal for further analysis, a low-pass Chebyshev Type I filter was used with an order of 8 , and the sampling rate of the data wase subsequently reduced by a factor of 25.

Linear deconvolution was used to estimate how the pupil responded to task events. Deconvolution analysis is a form of regression often used in fMRI analyses where physiological responses to fast stimulus presentations from each trial can introduce noise into the signal for an event of interest (Glover, 1999; McCloy et al., 2016). Therefore, to "deconvolve" the impulse response function (IRF) of the pupillary response to a given event, the pupil time-series data are multiplied by the pseudoinverse of the design matrix with the events of interest (Gardner et al., 2008). For each participant, the pupil's IRF was deconvolved to a letter repeat preceding a hit, a letter repeat preceding a miss, and the $1 \mathrm{~s}$ preceding a false alarm (FA), separately for each task condition (no distractors vs distractors). A single deconvolution block matrix was used, composed of three concatenated design matrices, one per event type, to covary out the other predictors in each IRF's estimation. The IRF was prespecified to be $4 \mathrm{~s}$ in duration, before the described analyses of the pupillometry data, on the basis of prior work examining the pupillary response as a proxy for LC activity on tasks demanding attention (Eldar et al., 2013, 2016). On inspection of the data collected in this study, this IRF length was deemed appropriate, although further analyses were subsequently conducted specifying several additional IRF lengths $(2.5,3,3.5,4.5$, and $5 \mathrm{~s}$ ) to best understand under what parameters group and task conditions differed. The amplitude of the pupil response was calculated as the median absolute deviation (MAD) of the IRF. If a participant's pupil response amplitude value was $>3$ or $<3$ SD from the mean of the pupil amplitudes of all participants in a group (by diagnosis), for a condition (presence vs absence of distractors), for an event (hit, FA, or miss), that value was assumed to be artifactual, treated as an outlier, and discarded. The threshold of 3 SDs for outlier detection accounts for both the designated $\alpha$ criterion of 0.05 used in all analyses and the sample size of each participant group (Cousineau and Chartier, 2010). Additionally, in a separate analysis, a deconvolution block matrix was used, with a single design matrix with the onset of distractor tones, to generate IRFs (also 4 $s$ in duration) for pupillary responses to distractors.

\section{Inferential analyses}

All inferential statistics were performed with $\mathrm{R}$ version 3.5.2 ( $\mathrm{R}$ Foundations for Statistical Computing), using the dplyr (Wickham et al., 2019a), psych (Revelle, 2019), effsize (Torchiano, 2019), lme4 (Bates et al., 2019), lmerTest (Kuznetsova et al., 2019), EMATools (Kleiman, 2017), and multcomp (Hothorn et al., 2019) packages. Figures were generated using the seaborn Python package (Waskom et al., 2018) and ggplot2 (Wickham et al., 2019b).

For analyses on sensitivity index $\left(d^{\prime}\right)$, criterion $(\mathrm{C})$, reaction time (RT), and pupil amplitude response (each to hits, FAs, and misses), linear mixed models were fitted to predict these variables, with group and task condition as fixed effect predictors and participant as a random effect predictor. For analyses on average baseline pupil size (for which there is only one derived measurement per participant), linear models were fitted to predict these variables, with group as a predictor. In addition to verifying the findings from the linear mixed models predicting pupil response amplitude to hits, FAs, and misses, for each such task event, the ratio of the pupil response amplitude in the presence of distractors to that in the absence of distractors was computed for each participant. For each task event, a linear model was fitted with this ratio as the dependent measure and group as the predictor. Because more than half of participants with ASD reported taking adrenergic-related medications, separately, models were fitted to predict each aforementioned dependent measure, with adrenergic-related medication use and task condition as fixed effect predictors and participant as a random effect predictor (for repeated measures). These models were fitted using the data from participants with ASD only, as only a single control participant reported using adrenergic-related medications.

Furthermore, to assess whether pupillometry measurements were associated with clinical metrics collected from participants with ASD, models were fitted to predict dependent pupillometry measurements with clinical scores as predictors. The ADOS scores for Language and Communication (LaCo), Reciprocal Social Interaction (RSI), and Restricted and Repetitive Behaviors (RRB), as well as task condition (absence versus presence of distractors), were used as fixed effector predictors, and participant was used as a random effect predictor (for repeated measures). These models were fitted using the data from participants with ASD only, as controls were not assessed on these clinical measures.

Absolute values of test statistics and effect sizes are reported. The $\alpha$ criterion for statistical significance was designated as 0.05 for all inferential statistical analyses. Cohen's $d$ was computed as an estimate of effect size (Fritz et al., 2012), as the magnitude of effect sizes is limited in this population of participants and can vary based on individual differences in the eye-tracker and experimental setup. Furthermore, in cases in which there was no statistical significance, to provide further evidence in support of the null hypothesis, an approximation for the Bayes factor 
a

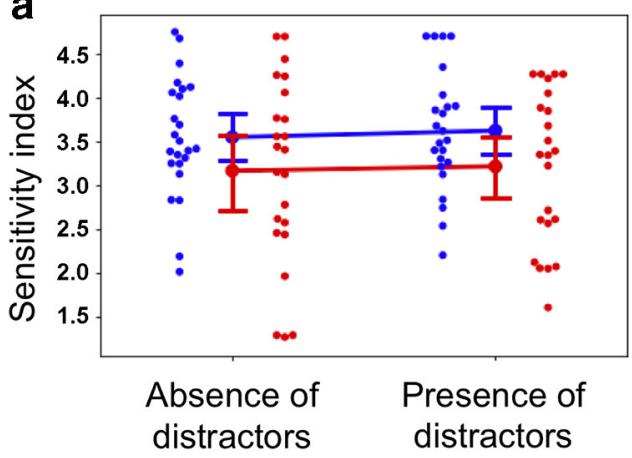

b

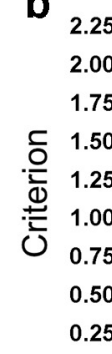

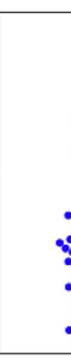

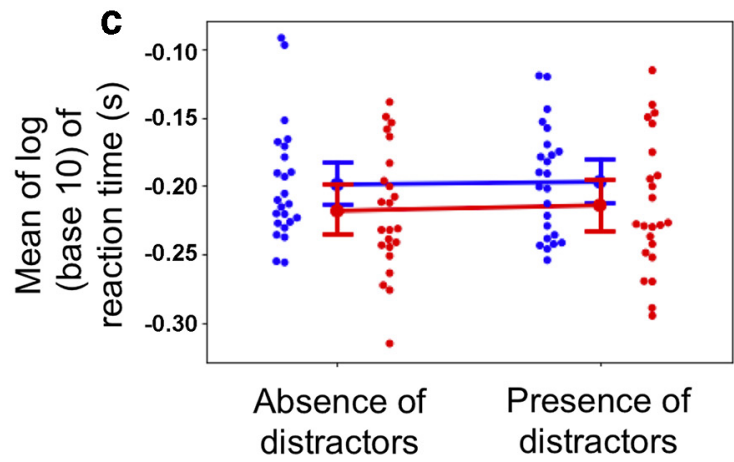

\section{distractors distractors}

Figure 2. Behavioral performance on the letter detection task: $(\boldsymbol{a}) d^{\prime},(\boldsymbol{b}) \mathrm{C}$, and (c) mean RT, across group (autism vs control) and condition (absence vs presence of distractor stimuli). Each point represents an individual participant. Line plots represent the bootstrapped $95 \% \mathrm{Cl}$ around the mean.

(BF) was computed using the respective Bayesian information criteria of the null model (excluding all fixed effect predictors) and alternative model (including all fixed effect predictors). A BF between 3 and 20, between 20 and 150 , or $>150$ was designated as positive, strong, or very strong evidence for the null hypothesis, respectively (Wagenmakers, 2007). Some participants do not have select data values (such as a participant who does not commit any FAs, and therefore has no pupil amplitude response to FAs); degrees of freedom (df), however, are reported for all inferential analyses.

\section{Classification analyses}

To validate the inferential statistical analyses, classification analyses were used to assess whether group membership could be predicted from pupil response amplitude. A logistic regression model was fitted with group as the dependent measure and the absolute difference of the pupil response amplitude between the two conditions (absence vs presence of distractors) as the predictor, for each event type (hit, FA, or miss). The LogisticRegression class within the scikit-learn version 19.1 (Pedregosa et al., 2011) package in Python version 3.7.1 (Python Software Foundation) was used with the saga solver and no regularization. Twenty repeats of fivefold cross-validations were performed to compute the predictive accuracy of group for each event and condition combination. A null distribution was created by shuffling the labels 10,000 times and performing the same cross-validation classification approach. The statistical significance ( $p$ value) of the classification accuracy was determined by a comparison to the null distribution, as (1-percentile), where percentile indexes the percentile of the true classification accuracy in the distribution of null distribution classification accuracies.

As three independent classification analyses were performed ( 3 event types), for these analyses, an accuracy value was considered significant if the $p$ value was lower than the Bonferroni-corrected criterion: 0.05/ $3=0.02$.

\section{Code accessibility}

All raw data are publicly available on Carnegie Mellon University's data repository, KiltHub, which is supplied by figshare. Experiment and

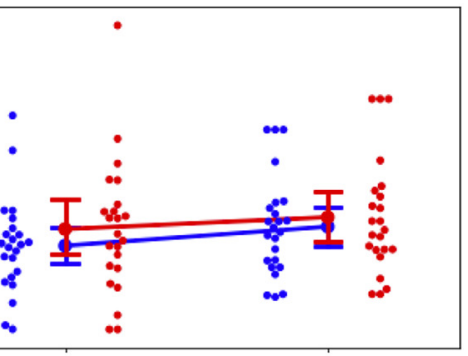

Absence of distractors
Presence of distractors
- control

- autism preprocessing MATLAB scripts, $\mathrm{R}$ and Python analysis code, and preprocessed data are available on GitHub (https://github.com/ michaelgrano/ASD_nback).

\section{Results}

Comparable between-group task performance in the absence and presence of distractor stimuli

Group differences in behavioral performance were initially analyzed as any such differences could confound observed group differences in pupillary responses. The $d^{\prime}$, $\mathrm{C}$, and mean RT were computed for each participant, for each task condition (absence vs presence of distractors). (If a participant's $d^{\prime}$ or $\mathrm{C}$ was positive or negative infinity, the maximum or minimum value for that participant's group in the given condition was substituted for these analyses, respectively.) Figure 2 shows the $d^{\prime}, \mathrm{C}$, and mean RT for the two groups. There was no significant effect of group $\left(t_{(56.29)}=1.57, p=0.12, d=0.42\right)$, task condition $\left(t_{(45.00)}=0.66\right.$, $p=0.52, d=0.20)$, or their interaction $\left(t_{(45.00)}=0.16, p=0.88\right.$, $d=0.05$ ) on $d^{\prime}$ (Fig. 2a). Likewise, there was no significant effect of group $\left(t_{(66.31)}=1.02, p=0.31, d=0.25\right)$, task condition $\left(t_{(45.00)}=1.86\right.$, $p=0.07, d=0.55)$, or their interaction $\left(t_{(45.00)}=0.49, p=0.62\right.$, $d=0.15$ ) on $\mathrm{C}$ (Fig. 2b). There was very strong evidence that neither group nor presence of distractor stimuli predicted $d^{\prime}(\mathrm{BF}=3262.08)$ or $\mathrm{C}(\mathrm{BF}=8760.19)$.

The mean RT (time between the onset of a letter repeat and a correct button press) across all correct responses was also computed for each participant, separately for each task condition. A log (base 10) transformation was applied to the raw RT data to account for the possibility of skewed or bimodal RT distributions (Whelan, 2008). There was no significant effect of group $\left(t_{(53.02)}=1.48, p=0.14, d=0.41\right)$ or task condition $\left(t_{(45.00)}=0.39\right.$, $p=0.70, d=0.11)$ on mean RT. There was also no significant interaction of group $\times$ task condition on mean $\mathrm{RT}\left(t_{(45.00)}=0.28\right.$, $p=0.78, d=0.08)$, and there was very strong evidence in favor of the null hypothesis $(\mathrm{BF}=39910754.61$; Fig. $2 c)$.

The effects of adrenergic-related medication use among participants with ASD on behavioral measures were also examined. There was no significant effect of medication use $\left(t_{(25.72)}=0.73\right.$, $p=0.47, d=0.29)$ or interaction of medication use $\times$ task condition $\left(t_{(21.00)}=1.08, p=0.29, d=0.47\right)$ on $d^{\prime}$. There was also no significant effect of medication use $\left(t_{(29.96)}=0.16, p=0.87\right.$, $d=0.06)$ or interaction of medication use $\times$ task condition $\left(t_{(21.00)}=1.35, p=0.19, d=0.59\right)$ on C. Likewise, there was no significant effect of medication use $\left(t_{(25.31)}=0.53, p=0.60, d=0.21\right)$ or interaction of medication use $\times$ task condition $\left(t_{(21.00)}=0.27\right.$, $p=0.79, d=0.12$ ) on mean RT. There was very strong evidence that medication use, task condition, and their interaction 


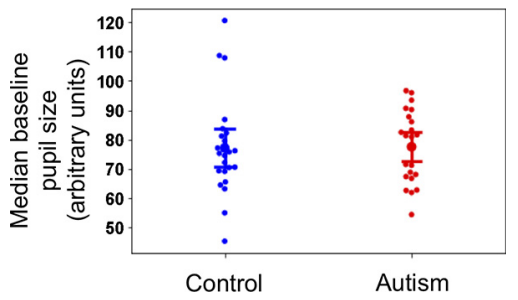

Figure 3. Median baseline pupil size of participants by group. Each point represents an individual participant. Line plots represent the bootstrapped $95 \% \mathrm{Cl}$ around the mean.

together did not predict $d^{\prime}(\mathrm{BF}=713.23), \mathrm{C}(\mathrm{BF}=2451.16)$, or mean RT $(B F=9425202.72)$.

Given the lack of a group difference on task performance, any differences in pupil size are unlikely to be attributed to differences in behavioral performance and, indeed, a simple task was selected specifically to equate performance as much as possible. The lack of an interaction between group $\times$ condition also rules out a foundational difference in working memory, a required component of the task, in the participants with autism versus controls. Finally, the lack of an effect of adrenergic-related medication use on $d^{\prime}, \mathrm{C}$, or mean RT among participants with ASD indicates that this potential confound is unlikely to have affected behavioral performance.

\section{No between-group differences in baseline pupil size}

Group differences in time-averaged pupil size were analyzed to rule out the possibility of any systematic a priori differences in pupil size between the groups. Baseline pupil size (recorded before each task block) was compared between groups to determine whether measurements of pupil size differed between participants with ASD and controls, independent of the letter detection task. As shown in Figure 3, there was no significant effect of group on the median baseline pupil size $\left(t_{(44)}=0.09\right.$, $p=0.93, d=0.03$ ), with positive evidence that baseline pupil size does not predict this measure $(\mathrm{BF}=6.75)$. Moreover, among participants with ASD, there was no significant effect of adrenergicrelated medications on median baseline pupil size $\left(t_{(20)}=0.84\right.$, $p=0.41, d=0.36$ ), with positive evidence that baseline pupil size does not predict this measure $(\mathrm{BF}=3.19)$. The lack of a main effect of group or of medication use indicates that there were likely no systematic differences in pupil size, thereby ruling out confounding variables that would be independent of the task.

\section{Individuals with ASD exhibited smaller task-evoked pupil response amplitudes than did controls in the presence but not absence of distractor stimuli}

Linear deconvolution analysis (Glover, 1999; McCloy et al., 2016) was used to approximate a 4 s IRF to each task event (hits, FAs, and misses) for each participant in each task condition. The individual IRFs of participants' pupillary responses to hits are shown in Figure 4. The pupil response amplitude was calculated as the MAD of the IRF, as this value captures the dispersion of the pupillary response, while reducing the impact of noise caused by limited data (Kret and Sjak-Shie, 2019). This is similar to the approach extensively adopted in the fMRI literature, where the dispersion of the BOLD signal time course has been used as a nonparametric measure of response amplitude (Power et al., 2018).

Between-group comparisons of the pupil amplitude response to each task event associated with a decision (hits and FAs) for each task condition (absence vs presence of distractors) were analyzed. These pupillary responses should reflect changes in LC activity because pupil dilations occur specifically in response to the appearance of a stimulus on a cognitive task (here, the oneback letter detection task) that results in a decision (here, a key press) (Aston-Jones and Cohen, 2005).

As evident from Figure 5, there was a significant interaction between group and the presence/absence of distractor stimuli on pupil amplitude in response to both hits $\left(t_{(43.63)}=3.06, p<0.01\right.$, $d=0.93)$ and FAs $\left(t_{(42.44)}=2.65, p=0.01, d=0.81\right)$. Furthermore, in the presence versus absence of distractor stimuli, there was a significant increase in pupil amplitude in response to hits $\left(t_{(43.28)}=2.93, p<0.01, d=0.89\right)$, independent of group, but no significant difference in response to FAs $\left(t_{(42.78)}=1.13, p=0.27\right.$, $d=0.34$ ). Moreover, there was no significant effect of group on pupil amplitude in response to either hits $\left(t_{(67.36)}=0.08, p=0.94\right.$, $d=0.02)$ or FAs $\left(t_{(70.46)}=0.17, p=0.87, d=0.04\right)$.

Post hoc contrast tests of the effect of task condition on pupil response amplitude performed separately for each group showed that, as anticipated (Gilzenrat et al., 2010), among controls, the pupil amplitude in response to hits was significantly higher in the presence versus absence of distractors $(z=2.93, p<0.01$, $d=0.61$ ). Notably, there was no such significant difference in response to hits among participants with $\operatorname{ASD}(z=1.43, p=0.15$, $d=0.31$ ). Moreover, while there was no significant betweengroup difference in pupil amplitude in response to hits in the absence of distractors $(z=0.08, p=0.94, d=0.02)$, in the presence of distractors, individuals with ASD exhibited lower pupil response amplitudes than did the controls $(z=2.80, p<0.01$, $d=0.98)$. Indeed, the ratio of the pupil response amplitude in the presence of distractors to that in the absence of distractors was significantly higher among controls than it was among participants with $\operatorname{ASD}\left(t_{(45.00)}=3.10, p<0.01, d=0.91\right.$; Fig. $\left.5 a\right)$.

Furthermore, among individuals with ASD, the pupil amplitude in response to FAs was significantly lower in the presence versus absence of distractors $(z=2.61, p<0.01, d=0.67)$, while this was not the case among controls $(z=1.13, p=0.26, d=0.24)$. As was the case with hits, the pupil amplitudes in response to FAs were not different between groups in the absence of distractors $(z=0.17, p=0.87, d=0.06)$, but in the presence of distractors, individuals with ASD exhibited lower pupil response amplitudes than controls $(z=2.57, p=0.01, d=0.75)$. Additionally, the ratio of the pupil response amplitude in the presence of distractors to that in the absence of distractors was significantly higher among controls than it was among participants with $\operatorname{ASD}\left(t_{(42.00)}=2.85\right.$, $p=0.01, d=0.87$; Fig. $5 b$ ).

The effects of medication use among participants with ASD on pupil amplitude in response to hits and FAs were also examined. There was no significant effect of medication use on pupil amplitude in response to either hits $\left(t_{(31.57)}=1.95, p=0.06\right.$, $d=0.69)$ or FAs $\left(t_{(33.69)}=1.13, p=0.27, d=0.39\right)$. Importantly, there also was no significant medication use $\times$ task condition interaction effect on pupil amplitude in response to either hits $\left(t_{(18.96)}=1.27, p=0.22, d=0.58\right)$ or FAs $\left(t_{(18.95)}=1.47, p=0.16\right.$, $d=0.67)$. There was very strong evidence that medication use, task condition, and their interaction together did not predict pupil amplitude in response to either hits $(\mathrm{BF}=3462709457.20)$ or FAs (BF = 233732511.49).

A $4 \mathrm{~s}$ IRF duration was decided a priori on the basis of prior work studying pupillary responses in the context of attention and decision-making (Eldar et al., 2013, 2016). To explore the group differences as a function of IRF, we examined pupil amplitude responses to hits and FAs. While the group $\times$ condition interaction on pupil response amplitudes to hits was insignificant 
a

Participants with ASD (absence of distractors)

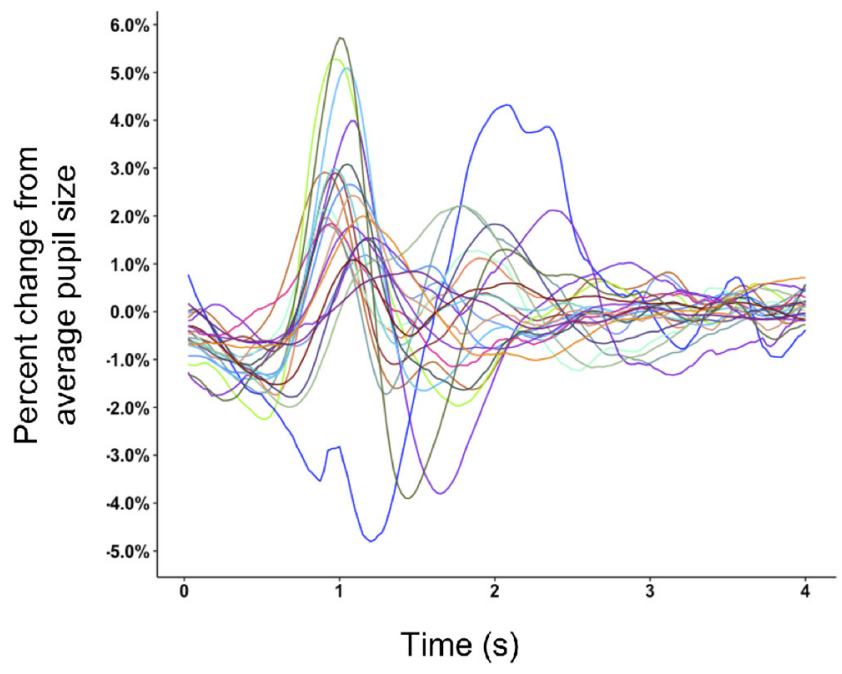

b
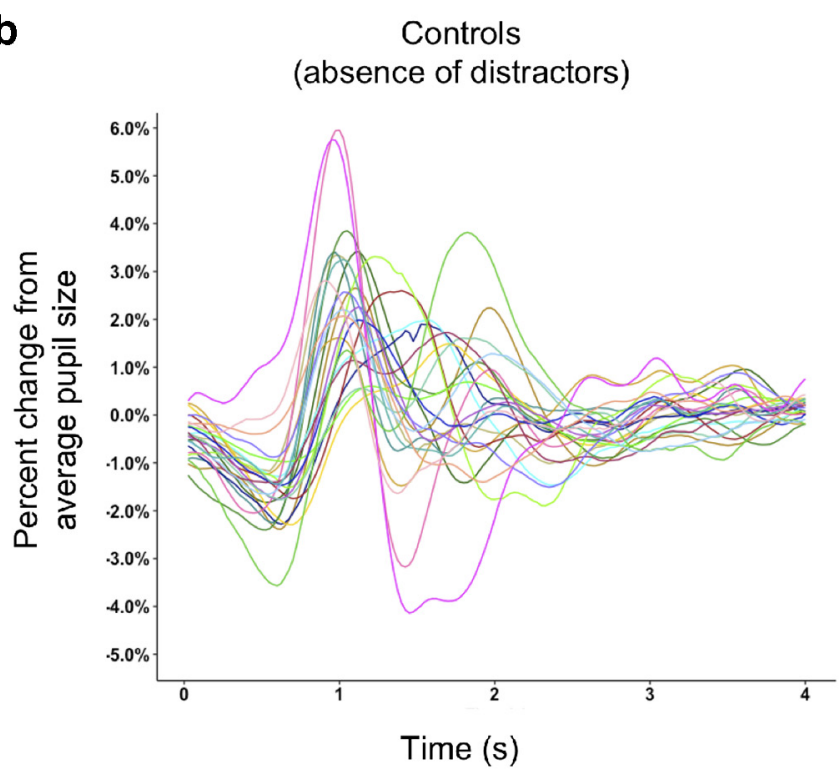

Participants with ASD (presence of distractors)
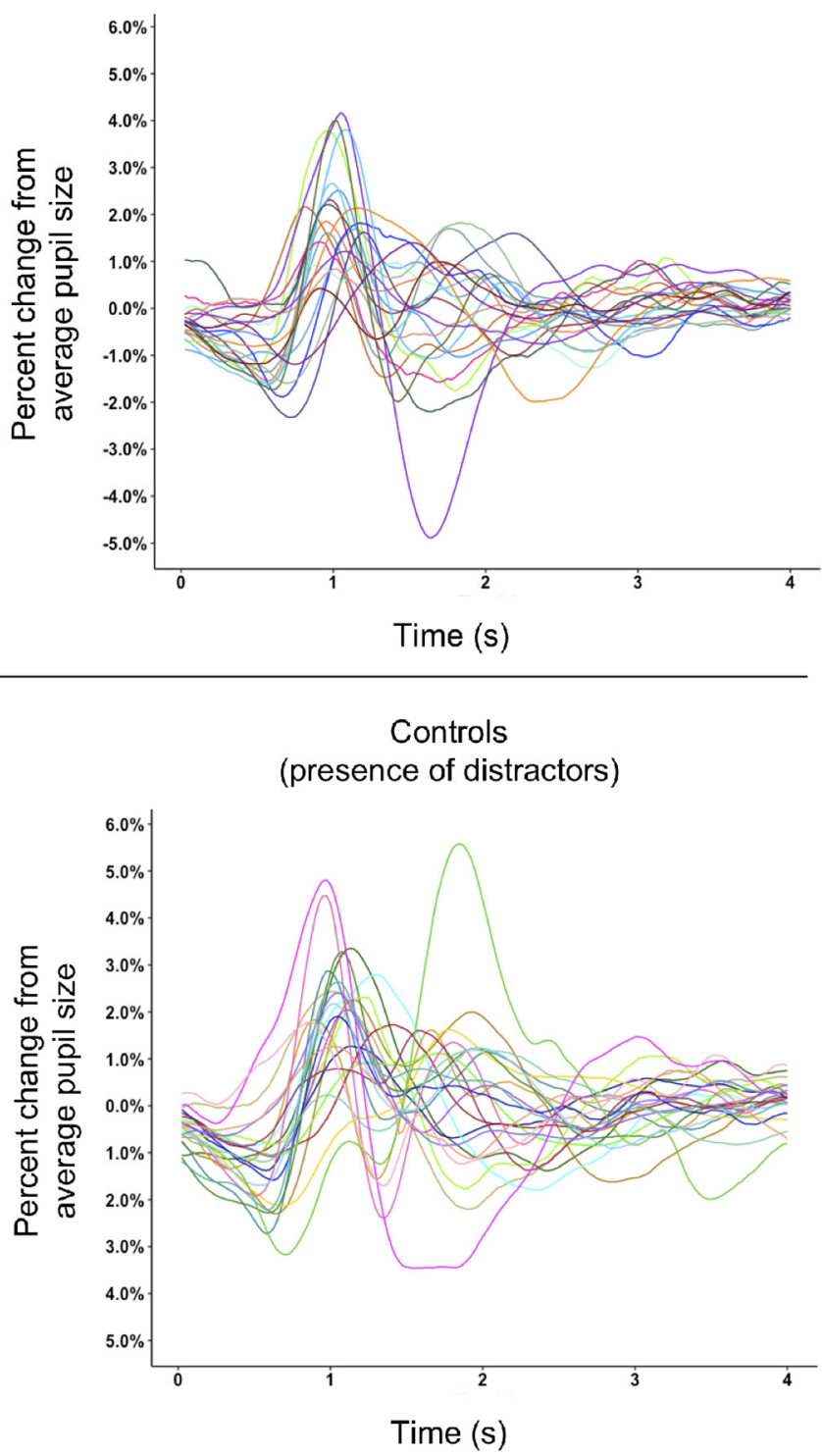

Figure 4. All participants' individual IRFs of pupillary responses to hits. Each color represents the IRF of a unique participant in the (a) ASD and (b) control groups. Left, Results in the absence of distractors. Right, Results in the presence of distractors.

at IRFs of $2.5 \mathrm{~s} \quad\left(t_{(44.43)}=0.67, p=0.51, d=0.20\right), 3 \mathrm{~s}$ $\left(t_{(43.85)}=1.59, p=0.12, d=0.48\right)$, and $3.5 \mathrm{~s}\left(t_{(42.65)}=1.72, p=0.09\right.$, $d=0.53)$, it was significant at an IRF of $4.5 \mathrm{~s}\left(t_{(43.25)}=3.07\right.$, $p<0.01, d=0.93)$, as reported previously, and $5 \mathrm{~s}\left(t_{(43.22)}=2.55\right.$, $p=0.01, d=0.77)$. Likewise, while the group $\times$ condition interaction to FAs was insignificant at an IRF of $3 \mathrm{~s}\left(t_{(43.91)}=1.58\right.$, $p=0.12, d=0.48)$ and $3.5 \mathrm{~s}\left(t_{(42.83)}=1.55, p=0.13, d=0.47\right)$, it was significant at an IRF of $2.5 \mathrm{~s}\left(t_{(42.12)}=2.28, p=0.03, d=0.70\right)$, $4.5 \mathrm{~s}\left(t_{(40.53)}=3.48, p<0.01, d=1.09\right)$, and $5 \mathrm{~s}\left(t_{(40.74)}=3.15\right.$, $p<0.01, d=0.99)$. Together, this indicates that, primarily, the differential group $\times$ task condition on pupil response amplitudes was best detected on IRFs of $4 \mathrm{~s}$ durations or longer. It is possible that changes in LC activity in response to the stimulus presentation and task decision require $4 \mathrm{~s}$ to evoke a corresponding pupil dilation.

Thus, overall, pupillary responses to stimuli that elicit behavioral reports (thereby suggestive of LC activity) (Aston-Jones and Cohen, 2005) in the distractor-present condition (i.e., with increased cognitive load and task engagement) were lower among individuals with ASD. However, in the absence of distractor stimuli, no between-group differences existed. These findings are unlikely to be attributed to differences in medication use between the two groups.

\section{No interaction effect between group and task condition on pupil amplitude responses to misses}

If the interaction effect between group and task condition on pupil response amplitude was specific to cognitive effort on the task (which would implicate LC activity) (Aston-Jones and Cohen, 2005), then we would not expect to see an interaction of group and task condition on pupil amplitude in response to misses (i.e., on trials where effort was likely to be least). Indeed, there was no significant interaction between group and task condition on pupil amplitude in response to misses $\left(t_{(44.00)}=1.41, p=0.17\right.$, $d=0.42$ ). Additionally, the ratio of the pupil response amplitude in the presence of distractors to that in their absence was not 
a

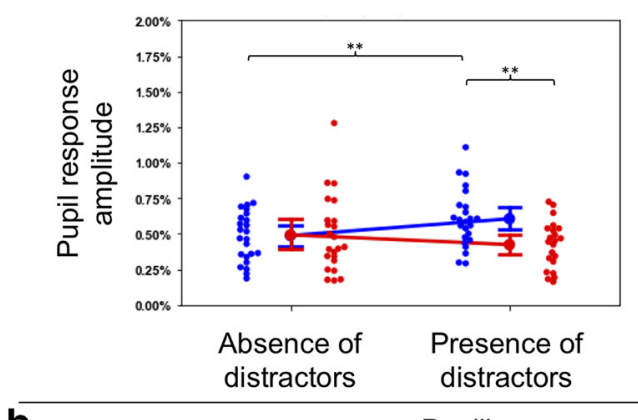

b

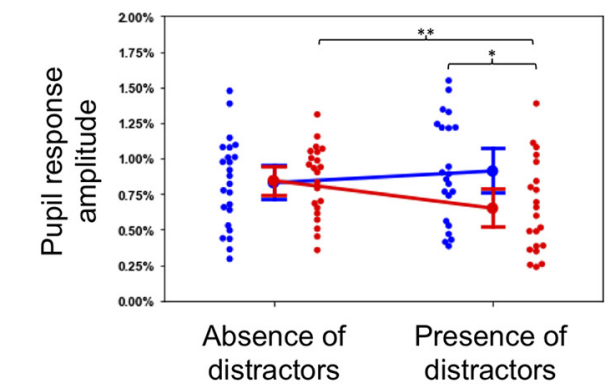

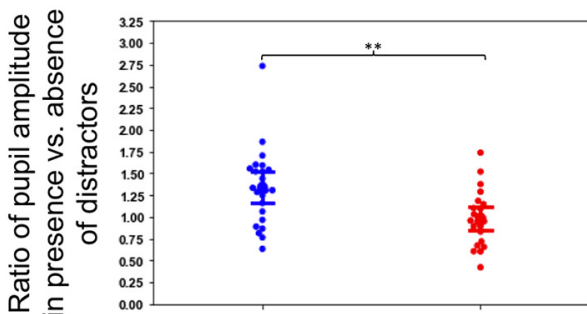

Control

Autism

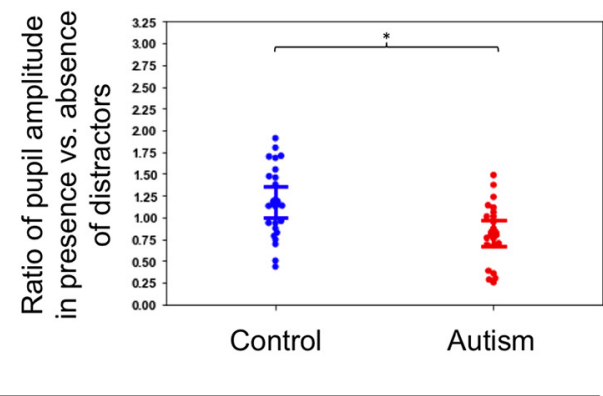

c

Pupillary response to misses
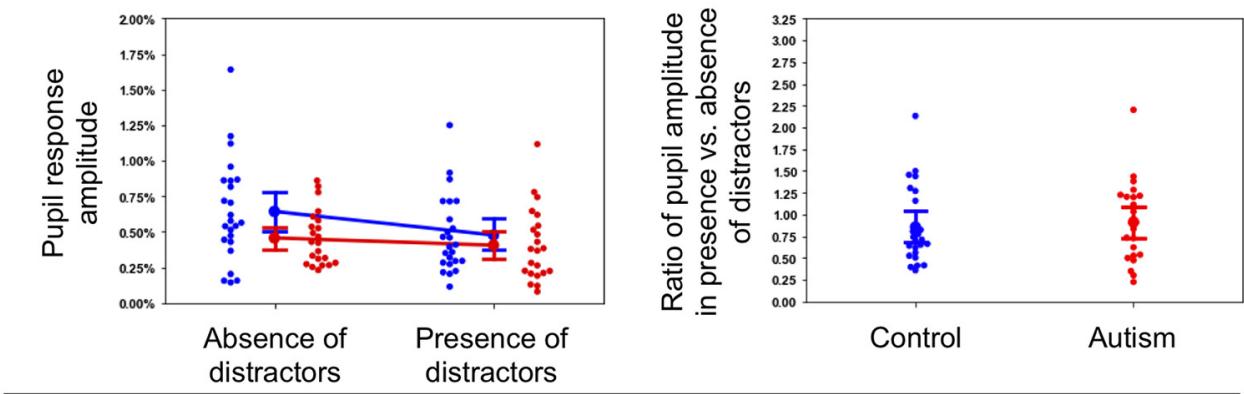

- control

- autism

Figure 5. Pupil response amplitudes to $(\boldsymbol{a})$ hits, $(\boldsymbol{b})$ FAs, and (c) misses, compared across groups and task conditions. Left panels, Pupil response amplitude, as defined as the MAD of the IRF of the respective pupil response, after normalization of the pupil time series data to the mean pupil size of the respective experiment block. Right panels, Ratio of the pupil response amplitude in the presence of distractors to that in the absence of distractors. Line plots represent the bootstrapped $95 \% \mathrm{Cl}$ around the mean. ${ }^{*} p<0.05$; ${ }^{* *} p<0.01$; for contrast tests.

significantly different between the two groups $\left(t_{(45.00)}=0.41\right.$, $p=0.68, d=0.12 ; \mathrm{BF}=6.21$, positive evidence for the null hypothesis). However, there were main effects of group and of task condition. Individuals with ASD exhibited lower pupil amplitudes in response to misses, independent of task condition, relative to controls $\left(t_{(70.72)}=2.28, p=0.03, d=0.54\right)$, and participants in both groups exhibited lower pupil amplitudes in response to misses in the presence versus absence of distractors $\left(t_{(44.00)}=\right.$ 2.92, $p<0.01, d=0.88$; Fig. $5 c$ ). It is conceivable that controls might have noticed misses across both task conditions more so than individuals with ASD, which might explain why controls' pupil amplitudes in response to misses were overall higher. Moreover, among participants with ASD, there was no significant effect of adrenergic-related medication use $\left(t_{(28.94)}=0.31\right.$, $p=0.76, d=0.12$ ) or medication use $\times$ task condition interaction $\left(t_{(20.00)}=2.06, p=0.05, d=0.92\right)$ on pupil amplitude in response to misses (although the effect size for the interaction is relatively prominent). There was very strong evidence that medication use, task condition, and their interaction did not predict pupil amplitude in response to misses $(\mathrm{BF}=3228110004.36)$.

Group membership can be predicted from the difference in pupil amplitude responses in the presence versus absence of distractors, only during hits and FAs (and not misses)

To validate the differential response to hits and FAs for the two groups and the effect of distractor condition, an assumption-free classification algorithm was used to determine whether group could be predicted from the task-evoked pupil response amplitudes alone. For each event type (hits, FAs, and misses), a logistic regression model was fitted to assess whether group classification (autism or control) could be predicted from the difference in the pupil response amplitude between the two conditions (absence vs presence of distractors). Consistent with the findings demonstrating an interaction between group and task condition on pupil response amplitudes to hits and FAs, group could be decoded from the between-conditions difference in pupil amplitude in 


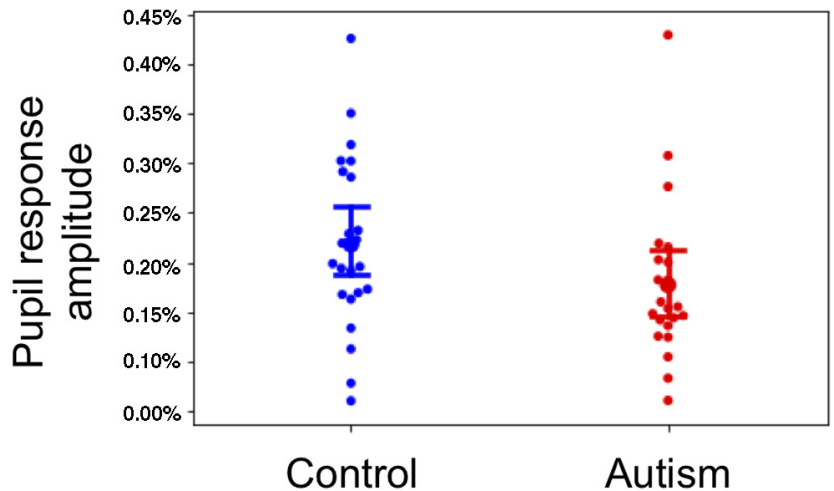

Figure 6. Pupil response amplitude to distractor stimuli, after normalization of the pupil time series data to the mean pupil size of the respective experiment block. Line plots represent the bootstrapped $95 \% \mathrm{Cl}$ around the mean. in response to auditory stimuli (Zekveld et al., 2018), LC activity is not associated with pupil dilations to task-irrelevant stimuli (Aston-Jones et al., 1999; Aston-Jones and Cohen, 2005; Gilzenrat et al., 2010), such as the onset of orthogonal distractors. To test this, pupil amplitude responses to the onset of distractor stimulus presentation were compared between the two groups. There was no significant effect of group on pupil response amplitude to the onset of distractors $\left(t_{(44)}=\right.$ 1.66, $p=0.10, d=0.49)$, suggesting that group does not predict pupillary response to distractors per se $(B F=1.68$; Fig. 6). Furthermore, among participants with ASD, there was no significant effect of adrenergic-related medication use $\left(t_{(20)}=\right.$ $0.01, p=0.99, d<0.01)$ on pupil response amplitude to distractors. Thus, group differences in pupillary dynamics are likely to be independent of pupil responses to the distractor stimulus presentations themselves. a

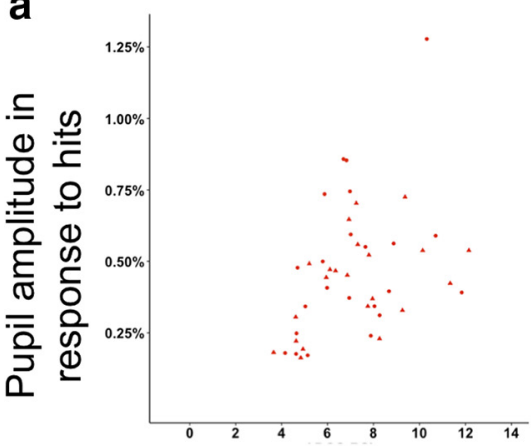

ADOS-RSI

C

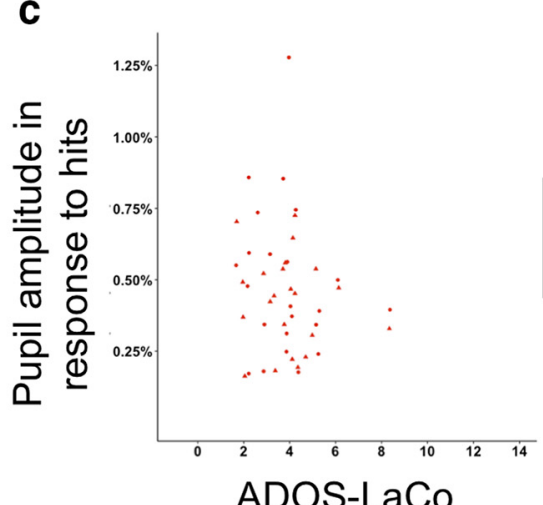

ADOS-LaCo

Figure 7. Relationship of pupil amplitude in response to hits versus (a) ADOS-RSI, (b) ADOS-RRB, and (c) ADOS-LaCo, among participants with ASD. Points are randomly jittered to avoid completely overlapping data.

response to hits (accuracy $=0.63, p<0.01)$ and FAs (accuracy $=0.56, p<0.01$ ) with above-chance accuracy. At the same time, the between-conditions difference in pupil amplitude in response to misses was not predictive of group and was below chance in accuracy (accuracy $=0.38, p=1.00$ ).

\section{No between-group differences of pupil dilations to the task-irrelevant distractor stimuli}

Group differences in pupil response amplitude just to distractor tone onsets were also assessed. While pupil dilations can occur

\section{Clinical metrics correlate with pupil response amplitude to hits}

ADOS scores (Lord et al., 1989) are considered to be the current "gold standard" assessment to quantify select behavioral features in ASD, although the clinical utility of this metric has been noted as problematic (Maddox et al., 2017; Lord et al., 2018). The ADOS score can be decomposed into three component scores: RSI, RRB, and LaCo (Lord et al., 1989). Pupil amplitude responses to hits were significantly lower for ASD participants with lower ADOS-RSI scores $\left(t_{(32.54)}=3.02, p<0.01, d=1.06\right.$; Fig. $\left.7 a\right)$ and higher ADOS-RRB scores $\left(t_{(32.54)}=\right.$ $2.05, p=0.05, d=0.72$; Fig. $7 b)$. There was, however, no significant effect of ADOSLaCo scores on pupil response amplitude to hits (although the negative effect size of ADOS-LaCo scores on the pupil response was prominent; $t_{(32.54)}=1.88, \quad p=0.07$, $d=0.66$; Fig. $7 c$ ). And importantly, there were no significant interaction effects of task condition with ADOS-RSI $\left(t_{(18.26)}=\right.$ $0.95, p=0.35, d=0.45)$, ADOS-RRB $\left(t_{(18.94)}\right.$ $=1.07, p=0.30, d=0.49$ ), or ADOS-LaCo $\left(t_{(19.13)}=0.66, p=0.52, d=0.30\right)$ scores. Thus, while there were no ADOS $\times$ task condition interactions, overall, the RSI and $\mathrm{RRB}$ components of the ADOS scores appear to be predictive of pupil response amplitude to hits among the participants with ASD.

On the other hand, there were no significant effects of ADOS-RSI $\left(t_{(31.10)}=\right.$ $1.61, p=0.12, d=0.58)$, ADOS-RRB $\left(t_{(31.10)}=0.33, p=0.75\right.$, $d=0.12)$, or ADOS-LaCo $\left(t_{(31.10)}=0.62, p=0.54, d=0.22\right)$ on pupil response amplitude to FAs. There also were no significant interaction effects of task condition with ADOS-RSI $\left(t_{(16.32)}=\right.$ $1.47, p=0.16, d=0.73)$, ADOS-RRB $\left(t_{(16.76)}=1.35, p=0.20\right.$, $d=0.66)$, or ADOS-LaCo $\left(t_{(16.32)}=0.85, p=0.41, d=0.42\right)$ scores on pupil response amplitude to FAs. Likewise, there were no significant effects of ADOS-RSI $\left(t_{(25.35)}=1.55, p=0.13, d=0.62\right)$, ADOS-RRB $\left(t_{(25.35)}=1.22, p=0.23, d=0.48\right)$, or ADOS-LaCo 
$\left(t_{(25.35)}=0.37, p=0.72, d=0.15\right)$ scores on pupil response amplitude to misses. And while there was a negative interaction effect of ADOS-RRB score and task condition on pupil response amplitude to misses $\left(t_{(18.00)}=2.67, p=0.02, d=1.26\right)$, there was no such interaction effect with ADOS-RSI $\left(t_{(18.00)}=1.27, p=0.22\right.$, $d=0.60)$ or ADOS-LaCo $\left(t_{(18.00)}=1.05, p=0.31, d=0.49\right)$ scores. Overall, there does not appear to be a clear relationship between ADOS scores and pupil response amplitude to FAs or misses, as there is for pupil response amplitude to hits, the most direct correlate of LC activity in the task paradigm.

\section{Discussion}

The goal of this study was to explore differences in LC activity (inferred with pupillometry) between individuals with ASD and matched controls performing a simple visual working memory task in the absence or presence of distractor tones. The ASD and control groups performed the task with statistically indistinguishable accuracy and speed, both in the presence and absence of distractors. However, specifically in the presence of distractors, individuals with ASD exhibited lower task-evoked pupil response amplitudes than did controls. Furthermore, group could be decoded with above-chance accuracy based solely on the difference of task-evoked pupil response amplitudes in the presence versus absence of distractors. This physiological effect could not be accounted for by medication use or baseline pupil size and was specific to task-evoked responses. As LC activity can be inferred from pupillary responses to task-relevant information, the lower task-evoked pupil response amplitudes in the ASD compared with control participants in the presence of distractors implicates dysregulation of LC activity.

Pupil dilations, specifically in association with task decisions, are established direct and indirect correlates of tonic and phasic LC activity, respectively. High tonic/low phasic LC activity is associated with increased neural gain, indiscriminately throughout cortex, thereby increasing neural responsivity. This indiscriminate increase in cortical gain is posited to increase behavioral flexibility, exploration of the task environment, and thus attention to both task-relevant and task-irrelevant stimuli. In contrast, when phasic responses weaken and global gain is reduced, attentional deployment shifts to task-relevant stimuli, and attention to task-irrelevant distractors becomes attenuated (Aston-Jones et al., 1999; Aston-Jones and Cohen, 2005; Gilzenrat et al., 2010; Pfeffer et al., 2018). It is thus particularly notable that, in the present study, participants with ASD evinced lower pupil response amplitudes in the presence of distractors because one would expect a typically developing individual to demonstrate increased pupil response amplitude (indicating higher phasic LC activity) under increased attentional demands (Aston-Jones and Cohen, 2005; Gilzenrat et al., 2010). This suggests an inflexibility in the regulation of LC activity in the participants with ASD.

A recent review from Bast et al. (2018) suggests that LC dysfunction might be associated with attentional differences in ASD, but there has been little prior empirical evidence to support this hypothesis. Several studies have shown phasic pupillary response differences in ASD (Martineau et al., 2011; Blaser et al., 2014; Nuske et al., 2014a,b; Krach et al., 2015; Lawson et al., 2017; Aguillon-Hernandez et al., 2019; Boxhoorn et al., 2019). However, in these studies, participants with ASD exhibited differences in task performance compared with controls; thus, differences in pupil dilations may be attributable to differences in task performance. Importantly, in the current study, participants with ASD and controls showed comparable task performance, on both signal detection theoretic and RT measures. A one-back task was specifically used because: (1) it was expected to elicit pupillary responses associated with task decisions; and (2) it was expected that ASD participants would perform comparably to controls (Williams et al., 2005). Had participants with ASD performed more poorly than controls, the observed interaction of group and task condition on pupil response amplitudes might have been a consequence of task performance rather than of LC activity per se. However, as task performance did not differ between the two groups, the between-group differences in taskevoked pupil response amplitudes across conditions suggest an inherent difference in LC physiology among the participants with ASD.

Furthermore, prior literature has suggested that individuals with ASD exhibit larger tonic pupil sizes (Anderson and Colombo, 2009; Anderson et al., 2013; Blaser et al., 2014). However, unlike prior findings that could be attributed to generalized autonomic arousal, the results of the present study indicate that differences in pupillary dynamics in the participants with ASD were specifically task-dependent and, therefore, provide clearer inference of LC activity per se. Group differences were noted, even after controlling for potential contributions of adrenergic-related medications, and although time-averaged pupil size recorded before each task block (which reflects individual variability in potential confounds, e.g., the eye-tracker setup and baseline autonomic levels) was equivalent between groups. Furthermore, an interaction of task condition and group on pupil response amplitudes was only revealed for phasic pupillary responses in association with hits and FAs, but not in association with misses. These are critical observations because LC activity has been correlated primarily with task-related decisions (AstonJones et al., 1999; Aston-Jones and Cohen, 2005; Gilzenrat et al., 2010). Thus, the effects in pupillary dynamics uncovered in this investigation are most likely to be associated with group differences, specifically in LC activity.

Generally, high tonic/low phasic LC activity is advantageous as it allows for exploratory behaviors and learning from new features of one's environment (Aston-Jones and Cohen, 2005; Gilzenrat et al., 2010). Studies in typically developing individuals suggest that, with high tonic/low phasic LC activity, individuals are more likely to attend selectively to salient stimulus cues (Eldar et al., 2016) or focus their attention on stimulus features to which they are individually predisposed to attend to (Eldar et al., 2013). However, an inability to regulate LC activity, and neural gain, could limit the ability to distinguish relevant versus irrelevant stimuli (Gilzenrat et al., 2010), thereby hampering the establishment of priors and the ability to learn from novel environmental input (Sinha et al., 2014; Dinstein et al., 2015). Thus, if individuals with ASD exhibit inflexibly elevated gain like the participants in this study, this could enhance attention to particular environmental stimuli, but would impair the ability to properly establish priors (Sinha et al., 2014). Attention might thus be deployed indiscriminately to task-relevant or task-irrelevant stimuli. This indiscriminate but selective attention from elevated gain could explain the fixated interests, selective attention, and exaggerated responsivity to sensory stimuli in individuals with ASD (Remington et al., 2009; Lord et al., 2018), as consistently high LC tonic activity would ultimately preclude the diversion of attention from distractor or task-irrelevant features in one's environment (Gilzenrat et al., 2010). If an individual with ASD cannot readily increase gain in the presence of distractors, this would significantly hamper typical learning processes. Indeed, 
while this study was designed to control for differences in behavioral performance, pupil response amplitudes to hits were nonetheless negatively and positively correlated with ADOS-RRB and ADOS-RSI scores, respectively. That is, the ASD participants with the highest gain might be expected to exhibit more repetitive behaviors and fixated interests. And interestingly, the ASD participants expected to exhibit challenges with social communication, an ASD feature not necessarily related to attention, appear to have opposite gain patterns. Thus, the differences in pupillary dynamics evinced in this study might be relevant for explaining canonical characteristics of ASD.

If individuals with ASD exhibit higher tonic/lower phasic LC activity than controls in an environment with both task-relevant and -irrelevant stimuli, such a dysregulation of the LC system would be consistent with the proposal of disrupted E-I homeostasis of cortical activity in ASD (Sur and Rubenstein, 2005; Rosenberg et al., 2015). Much research on E-I homeostasis has focused on the roles of glutamate and GABA in achieving this balance (Hensch, 2005; Samardzic et al., 2018), which is critical for efficient perceptual processing (Zhou and Yu, 2018). Indeed, there have been several demonstrations of atypical GABA activity in ASD (Pizzarelli and Cherubini, 2011; Robertson et al., 2016; Uzunova et al., 2016; Ajram et al., 2017). But perhaps the disruption in E-I homeostasis is not only or strictly a disruption in the ratio of excitatory to inhibitory activity, but in the gain, which is a measure of the simultaneous amplification (or dampening) of excitatory and inhibitory activity (Servan-Schreiber et al., 1990; Aston-Jones and Cohen, 2005; Hoshino, 2005; Pfeffer et al., 2018). Unregulated neural responsivity due to high tonic/ low phasic LC activity would, indeed, be consistent with findings that uncover highly variable neural responses to sensory stimuli in ASD (Dinstein et al., 2012; Haigh et al., 2015). In other words, with dysregulated gain in ASD from high tonic/low phasic LC activity, neural output would be highly unpredictable from neural input.

Consistently elevated tonic/depressed phasic LC activity, and consequently globally increased cortical gain, in an attentiondemanding environment is thus consistent with clinical and behavioral characteristics of ASD, as well as the E-I homeostasis disruption hypothesis. This study provides physiological evidence for an inherent difference in regulation of LC activity on a task on which individuals with ASD perform comparably to controls, laying the foundation for future work to explore the direct effects of this dysregulation on more challenging tasks that reflect the burdensome cognitive load of one's real-world environment. Clinically meaningful effect sizes for differences in pupillary dynamics still need to be explored. Also, it remains to be determined whether these results can be replicated across a wider spectrum of individuals with ASD: the IQ range of participants in this study was high, excellent for internal validity of the findings, but a limitation for their external validity. That said, these results nonetheless provide novel evidence for the LC's potential role in gain dysregulation, under varying attentional task demands, in ASD.

\section{References}

Abokyi S, Owusu-Mensah J, Osei KA (2017) Caffeine intake is associated with pupil dilation and enhanced accommodation. Eye 31:615-619.

Aguillon-Hernandez N, Mofid Y, Latinus M, Roché L, Bufo MR, Lemaire M, Malvy J, Martineau J, Wardak C, Bonnet-Brilhault F (2019) The pupil: a window on social automatic processing in autism spectrum disorder children. J Child Psychol Psychiatry. Advance online publication. Retrieved December 11, 2019. doi: 10.1111/jcpp.13170.

Ajram LA, Horder J, Mendez MA, Galanopoulos A, Brennan LP, Wichers RH, Robertson DM, Murphy CM, Zinkstok J, Ivin G, Heasman M, Meek D, Tricklebank MD, Barker GJ, Lythgoe DJ, Edden RA, Williams SC, Murphy DG, McAlonan GM (2017) Shifting brain inhibitory balance and connectivity of the prefrontal cortex of adults with autism spectrum disorder. Transl Psychiatry 7:e1137.

Anderson CJ, Colombo J (2009) Larger tonic pupil size in young children with autism spectrum disorder. Dev Psychobiol 51:207-211.

Anderson CJ, Colombo J, Unruh KE (2013) Pupil and salivary indicators of autonomic dysfunction in autism spectrum disorder. Dev Psychobiol 55:465-482.

Aston-Jones G, Cohen JD (2005) An integrative theory of locus coeruleusnorepinephrine function: adaptive gain and optimal performance. Annu Rev Neurosci 28:403-450.

Aston-Jones G, Rajkowski J, Cohen J (1999) Role of locus coeruleus in attention and behavioral flexibility. Biol Psychiatry 46:1309-1320.

Aston-Jones G, Rajkowski J, Kubiak P, Alexinsky T (1994) Locus coeruleus neurons in monkey are selectively activated by attended cues in a vigilance task. J Neurosci 14:4467-4480.

Babyak MA (2004) What you see may not be what you get: a brief, nontechnical introduction to overfitting in regression-type models. Psychosom Med 66:411-421.

Bast N, Pustka L, Freitag CM (2018) The locus coeruleus-norepinephrine system as pacemaker of attention - a developmental mechanism of derailed attentional function in autism spectrum disorder. Eur J Neurosci 47:115125.

Bates D, Maechler M, Bolker B, Walker S, Christensen RH, Singmann H, Dai B, Scheipl F, Grothendieck G, Green P, Fox J (2019) lme4: linear mixedeffects models using "Eigen" and S4. Available at https://CRAN.Rproject.org/package=lme4. Accessed May 25, 2019.

Blaser E, Eglington L, Carter AS, Kaldy Z (2014) Pupillometry reveals a mechanism for the autism spectrum disorder (ASD) advantage in visual tasks. Sci Rep 4:4301.

Boxhoorn S, Bast N, Supèr H, Polzer L, Cholemkery H, Freitag CM (2019) Pupil dilation during visuospatial orienting differentiates between autism spectrum disorder and attention-deficit/hyperactivity disorder. J Child Psychol Psychiatry. Advance online publication. Retrieved December 18, 2019. doi: 10.1111/jcpp.13179.

Brainard DH (1997) The Psychophysics Toolbox. Spat Vis 10:433-436.

Cousineau D, Chartier S (2010) Outliers detection and treatment: a review. Int J Psychol Res 3:58-67.

Dinstein I, Heeger DJ, Behrmann M (2015) Neural variability: friend or foe? Trends Cogn Sci 19:322-328.

Dinstein I, Heeger DJ, Lorenzi L, Minshew NJ, Malach R, Behrmann M (2012) Unreliable evoked responses in autism. Neuron 75:981-991.

Eldar E, Cohen JD, Niv Y (2013) The effects of neural gain on attention and learning. Nat Neurosci 16:1146-1153.

Eldar E, Niv Y, Cohen JD (2016) Do you see the forest or the tree? Neural gain and breadth versus focus in perceptual processing. Psychol Sci 27:1632-1643.

Fritz CO, Morris PE, Richler JJ (2012) Effect size estimates: current use, calculations, and interpretation. J Exp Psychol Gen 141:2-18.

Gardner JL, Merriam EP, Movshon JA, Heeger DJ (2008) Maps of visual space in human occipital cortex are retinotopic, not spatiotopic. J Neurosci 28:3988-3999.

Gilzenrat MS, Nieuwenhuis S, Jepma M, Cohen JD (2010) Pupil diameter tracks changes in control state predicted by the adaptive gain theory of locus coeruleus function. Cogn Affect Behav Neurosci 10:252-269.

Glover GH (1999) Deconvolution of impulse response in event-related BOLD fMRI. Neuroimage 9:416-429.

Haigh SM, Heeger DJ, Dinstein I, Minshew N, Behrmann M (2015) Cortical variability in the sensory-evoked response in autism. J Autism Dev Disord 45:1176-1190.

Hensch TK (2005) Critical period plasticity in local cortical circuits. Nat Rev Neurosci 6:877-888.

Hoshino O (2005) Cortical modulation of synaptic efficacies through norepinephrine. In: Adaptive and natural computing algorithms (Ribeiro B, Albrecht RF, Dobnikar A, Pearson DW, SteeleNC, eds), pp 70-73. Vienna: Springer. 
Hothorn T, Bretz F, Westfall P, Heiberger RM, Schuetzenmeister A, Scheibe S (2019) Multcomp: simultaneous Inference in general parametric models. Available at https://CRAN.R-project.org/package=multcomp. Accessed May 25, 2019.

Joshi S, Li Y, Kalwani RM, Gold JI (2016) Relationships between pupil diameter and neuronal activity in the locus coeruleus, colliculi, and cingulate cortex. Neuron 89:221-234.

Kaufman AS Lichtenberger EO (1999) Essentials of WAIS-III assessment. Hoboken, NJ: Wiley.

Kleiman E (2017) EMAtools: data management tools for real-time monitoring/ecological momentary assessment data. Available at https://CRAN.Rproject.org/package=EMAtools. Accessed January 25, 2020.

Krach S, Kamp-Becker I, Einhäuser W, Sommer J, Frässle S, Jansen A, Rademacher L, Müller-Pinzler L, Gazzola V, Paulus FM (2015) Evidence from pupillometry and fMRI indicates reduced neural response during vicarious social pain but not physical pain in autism. Hum Brain Mapp 36:4730-4744.

Kret ME, Sjak-Shie EE (2019) Preprocessing pupil size data: guidelines and code. Behav Res Methods 51:1336-1342.

Kuznetsova A, Brockhoff PB, Christensen RH (2019) lmerTest: tests in linear mixed effects models. Available at https://CRAN.R-project.org/package $=$ lmerTest. Accessed May 25, 2019.

Larsen RS, Waters J (2018) Neuromodulatory correlates of pupil dilation. Front Neural Circuits 12:21.

Lawson RP, Mathys C, Rees G (2017) Adults with autism overestimate the volatility of the sensory environment. Nat Neurosci 20:1293-1299.

Lord C, Elsabbagh M, Baird G, Veenstra-Vanderweele J (2018) Autism spectrum disorder. Lancet 392:508-520.

Lord C, Rutter M, Goode S, Heemsbergen J, Jordan H, Mawhood L, Schopler E (1989) Autism diagnostic observation schedule: a standardized observation of communicative and social behavior. J Autism Dev Disord 19:185212.

Maddox BB, Brodkin ES, Calkins ME, Shea K, Mullan K, Hostager J, Mandell DS, Miller JS (2017) The accuracy of the ADOS-2 in identifying autism among adults with complex psychiatric conditions J Autism Dev Disord 47:2703-2709.

Martineau J, Hernandez N, Hiebel L, Roché L, Metzger A, Bonnet-Brilhault F (2011) Can pupil size and pupil responses during visual scanning contribute to the diagnosis of autism spectrum disorder in children? J Psychiatr Res 45:1077-1082.

McCloy DR, Larson ED, Lau B, Lee AK (2016) Temporal alignment of pupillary response with stimulus events via deconvolution. J Acoust Soc Am 139:EL57-EL62.

McGuire RG (1992) Reporting of objective color measurements. HortSci 27:1254-1255

Nuske HJ, Vivanti G, Dissanayake C (2014a) Reactivity to fearful expressions of familiar and unfamiliar people in children with autism: an eye-tracking pupillometry study. J Neurodev Disord 6:14.

Nuske HJ, Vivanti G, Hudry K, Dissanayake C (2014b) Pupillometry reveals reduced unconscious emotional reactivity in autism. Biol Psychol 101:24-35.

Oldfield RC (1971) The assessment and analysis of handedness: the Edinburgh Inventory. Neuropsychologia 9:97-113.

Pedregosa F, Varoquaux G, Gramfort A, Michel V, Thirion B, Grisel O, Blondel M, Prettenhofer P, Weiss R, Dubourg V, Vanderplas J, Passos A, Cournapeau D, Brucher M, Perrot M, Duchesnay É (2011) Scikit-learn: machine learning in Python. J Mach Learn Res 12:2825-2830.

Pfeffer T, Avramiea AE, Nolte G, Engel AK, Linkenkaer-Hansen K, Donner TH (2018) Catecholamines alter the intrinsic variability of cortical population activity and perception. PLoS Biol 16:e2003453.
Pizzarelli R, Cherubini E (2011) Alterations of GABAergic signaling in autism spectrum disorders. Neural Plast 2011:297153.

Power JD, Plitt M, Gotts SJ, Kundu P, Voon V, Bandettini PA, Martin A (2018) Ridding fMRI data of motion-related influences: removal of signals with distinct spatial and physical bases in multiecho data. Proc Natl Acad Sci USA 115:E2105-E2114.

Ranganathan P, Pramesh CS, Buyse M (2016) Common pitfalls in statistical analysis: the perils of multiple testing. Perspect Clin Res 7:106-107.

Remington A, Swettenham J, Campbell R, Coleman M (2009) Selective attention and perceptual load in autism spectrum disorder. Psychol Sci 20: $1388-1393$.

Revelle W (2019) psych: procedures for psychological, psychometric, and personality research. Available at https://CRAN.R-project.org/package= psych. Accessed May 25, 2019.

Robertson CE, Kravitz DJ, Freyberg J, Baron-Cohen S, Baker CI (2013) Slower rate of binocular rivalry in autism. J Neurosci 33:16983-16991.

Robertson CE, Ratai EM, Kanwisher N (2016) Reduced GABAergic action in the autistic brain. Curr Biol 26:80-85.

Rosenberg A, Patterson JS, Angelaki DE (2015) A computational perspective on autism. Proc Natl Acad Sci USA 112:9158-9165.

Samardzic J, Jadzic D, Hencic B, Strac JJ (2018) GABA/glutamate balance: a key for normal brain functioning. In: GABA glutamate: new developments in neurotransmission research. IntechOpen:London, UK.

Servan-Schreiber D, Printz H, Cohen JD (1990) A network model of catecholamine effects: gain, signal-to-noise ratio, and behavior. Science 249: 892-895.

Sinha P, Kjelgaard MM, Gandhi TK, Tsourides K, Cardinaux AL, Pantazis D, Diamond SP, Held RM (2014) Autism as a disorder of prediction. Proc Natl Acad Sci USA 111:15220-15225.

Sur M, Rubenstein JLR (2005) Patterning and plasticity of the cerebral cortex. Science 310:805-810.

Torchiano M (2019) effsize: efficient effect size computation. Available at https://CRAN.R-project.org/package=effsize. Accessed January 25, 2020.

Urai AE, Braun A, Donner TH (2017) Pupil-linked arousal is driven by decision uncertainty and alters serial choice bias. Nat Commun 8:14637.

Uzunova G, Pallanti S, Hollander E (2016) Excitatory/inhibitory imbalance in autism spectrum disorders: implications for interventions and therapeutics. World J Biol Psychiatry 17:174-186.

Wagenmakers EJ (2007) A practical solution to the pervasive problems of $\mathrm{p}$ values. Psychon Bull Rev 14:779-804.

Waskom M, Botvinnik O, O'Kane D, Hobson P, Ostblom J, Lukauskas S, Gemperline DC, Augspurger T, Halchenko Y, Cole JB, Warmenhoven J, De Ruiter J, Pye C, Hoyer S, Vanderplas J, Villalba S, Kunter G, Quintero E, Bachant P, Martin M, et al. (2018) mwaskom/seaborn: v0. Zenodo 9:2018.

Whelan R (2008) Effective analysis of reaction time data. Psychol Rec $58: 475-482$

Wickham H, François R, Henry L, Müller K (2019a) dplyr: a grammar of data manipulation. Available at https://CRAN.R-project.org/package=dplyr. Accessed May 25, 2019.

Wickham H, Chang W, Henry L, Pedersen TL, Takahashi K, Wilke C, Woo K (2019b) ggplot2: create elegant data visualisations using the grammar of graphics. Available at https://CRAN.R-project.org/package=ggplot2. Accessed May 25, 2019.

Williams DL, Goldstein G, Carpenter PA, Minshew NJ (2005) Verbal and spatial working memory in autism. J Autism Dev Disord 35:747-756.

Zekveld AA, Koelewijn T, Kramer SE (2018) The pupil dilation response to auditory stimuli: current state of knowledge. Trends Hear 22:2331216518777174

Zhou S, Yu Y (2018) Synaptic E-I balance underlies efficient neural coding. Front Neurosci 12:46. 